\title{
Ultra-Thin TEM Sample Preparation with Advanced Backside FIB Milling Method
}

\author{
Hyo-jin Kang, Jong Hyeop Kim, Jang won Oh, Tae Sun Back, and Ho Joung Kim
}

Research and Development Division, Hynix Semiconductor Inc., San 136-1, Ami-ri, Bubal-eub, Ichon-si, Kyoungki-do 467-701, Korea

As semiconductors such as DRAM and NAND flash process geometries shrink, manufactures increasingly rely on cross-sectional Transmission Electron Microscopy (TEM) for monitoring the process, analyzing defects, and investigating interface layer morphology. Sample preparation is a critical step in TEM that significantly determines the quality of structural characterization and chemical analysis of the smallest and most critical structures. With advanced processes at $30 \mathrm{~nm}$ and below, the sample needs to be less than $20 \mathrm{~nm}$ in thickness in order to avoid overlap among small scale structures. Dual Beam Focused Ion Beam (FIB) systems have been widely used for TEM sample preparation, which allowed site specific sample preparation process with high throughput and precision. However, the inevitable FIB artifacts of the preparation process are curtaining and beam induced damage, which reduces the quality of the TEM imaging and limits the minimal useful specimen thickness. The curtaining artifacts occur where there are areas of different sputtering rates or voids within the specimen, for example a tungsten contact to silicon [1]. To eliminate curtaining artifacts, milling the sample from the side or the bottom has been shown to control curtaining very well [2-3]. Obtaining accurate, high quality thin sample free from damaged layer also requires low $\mathrm{kV}$ milling. The curtaining artifacts can be worse at low $\mathrm{kV}$.

In this paper, the uniform and damage-free TEM sample preparation with thickness less than $20 \mathrm{~nm}$ using advanced backside FIB milling method was successfully fabricated. The backside FIB milling method on FEI Helios 400S is described below.

FIG. 1 (a) shows the modified stub for backside FIB milling method. The use of the modified stub in the FIB has efficiency advantages over alternative methods because it allows fast and precise sample flipping of 180 degrees. Sample was lifted out of the bulk sample and attached in situ to the $2^{\text {nd }}$ probe (b-c). Holder was unloaded and the modified stub was flipped 180 degree in order to place the sample in a backside FIB milling orientation: bottom/substrate side up (towards the ion beam) after that the holder was loaded (e). Sample was detached from a $2^{\text {nd }}$ probe and later attached on $\mathrm{Cu}$ grid using $1^{\text {st }}$ probe (f-h), and 1st probe was separated with the sample. These steps are illustrated in FIG.1. After that milling away the bottom (substrate side) of the end of the sample using a high beam current to leave an approximately 1.5 um long, 1.5 um thick slab. And then, a platinum protection layer was deposited over the area of interest prior to ion milling, to protect the surface from ion damage. FIG.2. is shown SEM images of the sample without (a)/with (b) buffer layer. At $100 \mathrm{~nm}$ thickness, thinning at $30 \mathrm{kV}$ accelerating voltage was stopped to prevent the formation of an amorphous layer on both sidewalls of the sample. Finally, low $\mathrm{kV}$ cleaning was carried out at $5 \mathrm{kV}, 2$ $\mathrm{kV}, 1 \mathrm{kV}$ on both sides of the sample. For the final low $\mathrm{kV}$ cleaning $1 \mathrm{kV}$ and $14 \mathrm{pA}$ with an incidence angle of 5 degree is used. The thinning process can again be monitored using SEM. FIG. 3 shows TEM images of NAND flash sample by the conventional method (a) and the backside FIB milling method (b, c). In case of the sample with thickness less than $20 \mathrm{~nm}$, we could observe the image of without an overlap of Shallow Trench Isolation(STI). 
References

[1] Introduction to Focused Ion Beams, eds. L.A. Giannuzzi and F.A. Stevie, Springer (2005).

[2] Eduardo Montoya et al. Microscopy Research and Technique 6 (2007) 70 1060.

[3] Stephen M. Schwarz1 et al., Microsc. Microanal. 9 (Suppl. 2) (2003) 116.
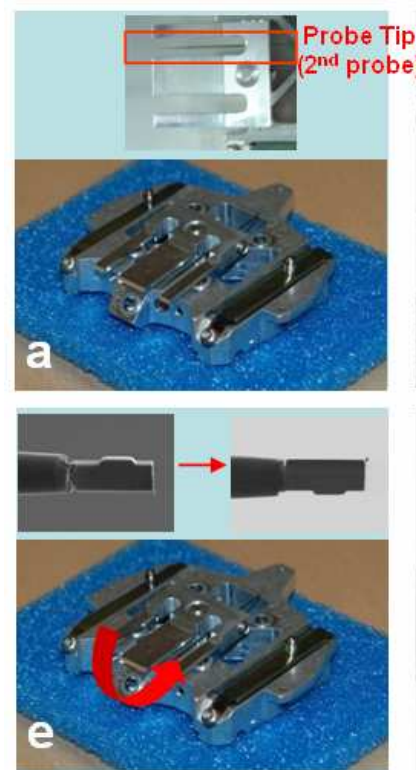
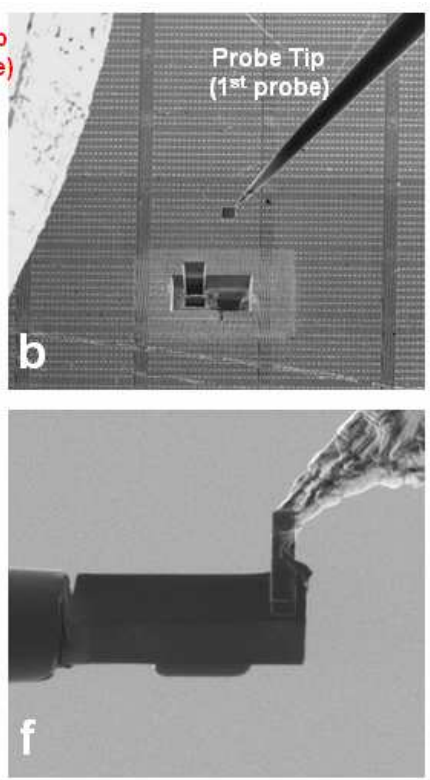
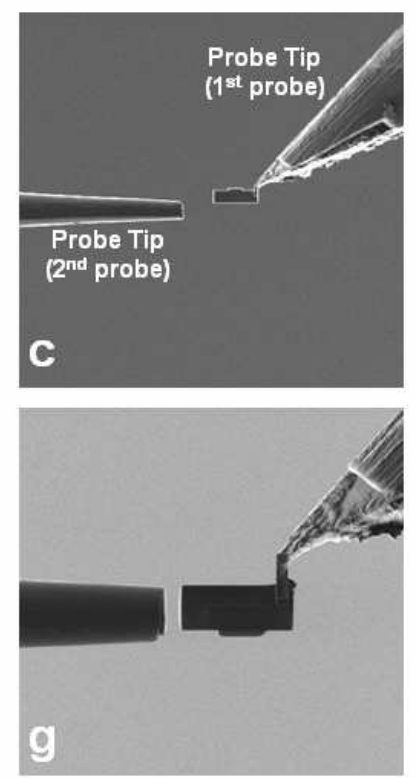
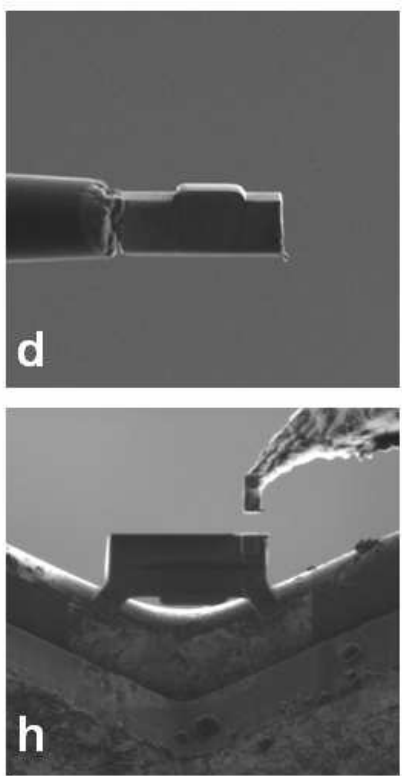

FIG. 1 Illustration of the backside milling method steps: (a) modified stub (b) extraction (c) $2^{\text {nd }}$ probe attach $(\mathrm{d}) 1^{\text {st }}$ probe detach (e) invert stub $180^{\circ}(\mathrm{f}) 1^{\text {st }}$ probe attach $(\mathrm{g}) 2^{\text {nd }}$ probe detach, and (h) attach on $\mathrm{Cu}$ grid using $1^{\text {st }}$ probe
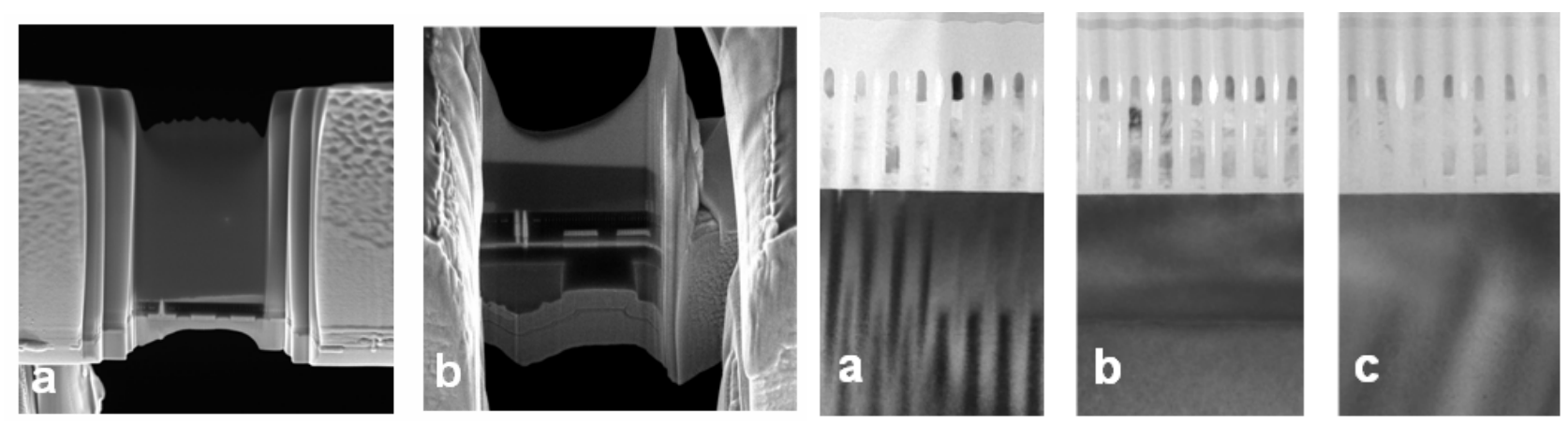

FIG. 2 SEM images of the sample without (a) /with(b) buffer layer
FIG. 3 TEM images of NAND flash sample by the conventional method (a) and backside milling method (b, c). Sample thickness is about $30 \mathrm{~nm}$ $(\mathrm{a}, \mathrm{b})$, and $20 \mathrm{~nm}(\mathrm{c})$, respectively 